\title{
Pediatric surgical site infection in the developing world: a Kenyan experience
}

\author{
James H. Wood • Peter M. Nthumba • \\ Edita Stepita-Poenaru · Dan Poenaru
}

Accepted: 19 January 2012/Published online: 2 February 2012

(C) Springer-Verlag 2012

\begin{abstract}
Background The purpose of the current study was to determine the incidence of pediatric surgical site infections (SSIs) at an academic children's hospital in rural subSaharan Africa and to identify potentially modifiable risk factors.

Methods Prospectively collected data from 1,008 surgical admissions to BethanyKids Kijabe Hospital (Kijabe, Kenya) were analyzed retrospectively. Follow-up data were available in 940 subjects.

Results SSIs occurred in $6.8 \%$ of included subjects $(N=64)$. Superficial $(69 \%)$ and deep $(29 \%)$ infections of the back (38\%) and head (25\%) were most common. When comparing children who developed SSI to those who did not, we found that wound contamination classification and duration of operation were the only variables with significant differences between groups.

Conclusions Our rate of SSI among pediatric patients in sub-Saharan Africa is the lowest reported in the literature to date. More work is needed to identify modifiable risk factors for pediatric SSI in low- and middle-income countries.
\end{abstract}

Presented at the 7th Congress of the Pan-African Paediatric Surgical Association, Accra, Ghana, 17-23 August 2008.

J. H. Wood ( $\bowtie)$

Department of Surgery, University of Colorado

Denver School of Medicine, 12631 East 17th Avenue,

Box C302, Aurora, CO 80045, USA

e-mail: james.wood@ucdenver.edu

P. M. Nthumba · E. Stepita-Poenaru · D. Poenaru

BethanyKids at Kijabe Hospital, Kijabe, Kenya
Keywords Surgical site infection - Rural surgery · Developing world

\section{Introduction}

While pediatric surgical site infections (SSIs) are increasingly rare in wealthy nations, they continue to be a major cause of morbidity and mortality in low- and middle-income countries (LMICs) [1-3]. Studies addressing this problem are sparse and geographically sporadic, and very little is known about the actual incidence of health-care-related infections among children in areas of the world such as Africa. This dearth of information is an impediment to the design and implementation of effective interventions to prevent SSIs among the most vulnerable children around the world. We therefore undertook the current study to determine the incidence of pediatric SSIs at our institution, an academic pediatric surgical center in rural Kenya, and to identify potentially modifiable risk factors for pediatric SSI relevant to LMICs.

\section{Methods}

Study design

Prospectively collected data for all children (age $<18$ years) who underwent operation at our institution between January and November 2007 were analyzed retrospectively. These data were collected as part of a cluster-randomized crossover trial (NCT00987402) evaluating surgeon hand scrubbing techniques (soap vs. alcohol rub) that has been published elsewhere [4]. Institutional ethics committee approval was obtained for this study, as were written consents for all included patients. 
Setting

Bethany Kids Kijabe Hospital is a 68-bed pediatric surgical unit within the AIC Kijabe Hospital, a 240-bed charitable general hospital in Kijabe, Kenya.

\section{Subject inclusion and exclusion}

All patients under the age of 18 years who underwent any operation at our hospital during the study period were assessed for inclusion in the study. Children undergoing reoperation (within 2 weeks of another surgical intervention) and children with operations classified as dirty were excluded from enrollment in the study.

\section{Variables and outcomes measured}

SSIs were diagnosed according to the modified US Centers for Disease Control and Prevention (CDC) definitions for nosocomial infection and were classified according to anatomical location (abdomen, groin, head, etc.) and depth (superficial incisional, deep incisional, or organ/ space) [5].

Potential SSIs were documented by a trained staff nurse, and final diagnosis was by consensus among the study collaborators. Patients were surveyed for signs of SSI on ward rounds 3-4 days per week, and patients discharged prior to completion of data collection were reviewed in the outpatient clinic or contacted by telephone. The follow-up period was defined as 30 days after the initial operation (or 1 year if surgical implants were used). Routine microbiological investigation of causative organisms was not performed as part of the study.

Major analyzed variables included patient demographics (age and gender), admission type, operative procedure, surgical specialty, American Society of Anesthesiologists (ASA) patient risk classification, surgical site contamination classification, and duration of operation.

\section{Data analysis}

Statistical tests of significance included ANOVA and Student's $t$ test for normal variables (reporting mean \pm standard error of the mean) and Mann-Whitney $U$ test for non-normal variables, including age and duration of operation (reporting median and interquartile range). Onesample Kolmogorov-Smirnov tests were used to determine variable normality. Categorical data were analyzed using Pearson's Chi-square or Fisher's exact test (with frequencies $<5$ for any included variable). All data were collected in Microsoft Access ${ }^{\circledR}$, and analyses were performed using IBM SPSS Statistics ${ }^{\circledR}$ software.

\section{Results}

A total of 1,008 children were enrolled in the study. Of those, valid SSI data with adequate follow-up were available for 940 children (93\% of enrolled subjects). SSIs occurred in 64 children $(6.8 \%)$ and included 45 superficial (69\%), 19 deep (29\%), and one organ/space (1.5\%) infection. The most common sites of SSI were the back $(38 \%)$ and head $(25 \%)$, followed by the abdomen, groin, and limbs (12.5\% each). SSIs were diagnosed on post-operative day $17 \pm 1.8$ days (range 5-60 days).

\section{Patient characteristics}

The study included 382 girls and 558 boys (59\%) with a median age of $1.2 \pm 4.8$ years (range $0-18$ years). When comparing children who developed SSI with those who did not, we found no significant differences in median age $(P=0.17)$ or gender $(P=0.99)$. The majority of the patients had an ASA classification of either $1(41 \%)$ or 2 $(55 \%)$. Four percent of patients were ASA class 3 and one patient was ASA class 4. SSI rates were 6, 7.6, 2.9, and $100 \%$ for ASA classes $1,2,3$, and 4 , respectively $(\eta=0.13)$.

Operative characteristics

Operations were categorized as neurosurgical (63\%), general $(14 \%)$, orthopedic $(9 \%)$, urological $(8 \%)$, plastic $(5.3 \%)$, and gynecological (1\%). SSIs occurred most commonly in general surgery cases $(11.6 \%)$, followed by neurosurgical $(7 \%)$ and plastic surgery $(6 \%)$ cases. Urological, orthopedic, and gynecological procedures had the lowest rates of SSI $(3.8,3.7$, and $0 \%$, respectively). However, these differences failed to reach statistical significance $\left(\chi^{2} P=0.15\right)$.

There were 887 elective operations (94\%) and 53 emergency operations, with no significant difference in the rate of SSI between groups (6.4 vs. $13 \%$, respectively, $\chi^{2}$ $P=0.12$ ). Likewise, there was no significant difference in the rates of SSI on comparing patients who had inpatient versus outpatient operations (6.8 vs. $8.3 \%$, Fisher's exact $P=0.58)$ or those who had general endotracheal anesthesia versus conscious sedation $\left(6.6\right.$ vs. $8.3 \%, \chi^{2}$ $P=0.47)$.

The median duration of operation was $50 \pm 45 \mathrm{~min}$. For patients who did not develop SSI, the median duration of operation was $50 \pm 46$ compared with $78 \pm 60 \mathrm{~min}$ for those who did develop SSI $(P=0.007)$.

Contamination and antibiotics

Surgical sites were classified as clean in $66 \%$, as cleancontaminated in $16 \%$, and as contaminated in $18 \%$ of 
Table 1 Linear regression analysis of risk factors for surgical site infection

\begin{tabular}{lcl}
\hline & Standardized $\beta$-coefficient & $P$ value \\
\hline Age & -0.04 & 0.34 \\
Gender & -0.003 & 0.93 \\
Admission type $^{\mathrm{a}}$ & -0.005 & 0.89 \\
Urgency of operation $^{\mathrm{b}}$ & -0.61 & 0.12 \\
Wound contamination $_{\text {Anesthesia type }}$ & 0.13 & $0.001^{*}$ \\
Surgical specialty & -0.04 & 0.34 \\
Duration of operation & 0.09 & 0.38 \\
Antibiotic prophylaxis & 0.27 & $0.03 *$ \\
\hline
\end{tabular}

* Significance set on $P<0.05$

${ }^{\text {a }}$ Inpatient versus outpatient

b Emergent versus elective

cases. SSI occurred in $4.4 \%$ of clean cases, $6.2 \%$ of cleancontaminated cases, and $17 \%$ of contaminated cases. $\left(\chi^{2}\right.$ $P<0.001)$. Peri-operative antibiotics were used in $85 \%$ of clean cases, $93 \%$ of clean-contaminated cases, and $99 \%$ of contaminated cases, at the operating surgeon's discretion $\left(\chi^{2} P<0.0001\right)$. SSI occurred in $8 \%$ of cases in which antibiotics were used compared with $4 \%$ of cases in which antibiotics were not used $\left(\chi^{2} P=0.19\right)$ No significant association of prophylactic antibiotic use and SSI was found when comparing within surgical site contamination groups.

\section{Multivariate analysis}

Linear regression of potential risk factors for SSI showed only surgical site contamination and duration of operation to be associated with a significant difference in rates of SSI. However, these variables were not strong predictors of SSI (contamination $\beta=0.13, P=0.001$; duration $\beta=0.09, P=0.03$ ) (see Table 1 ).

\section{Discussion}

Recent advances in peri-operative patient care have made SSIs rare, particularly in the USA and other high-income nations $[1,6,7]$. However, several reports suggest that SSIs occur much more commonly in developing nations, where prolonged hospitalization, higher treatment costs, and the potential long-term disability that characterize SSI pose a critical threat to the fragile economic and social conditions of many individuals and communities [3, 4, 8-11]. A recent meta-analysis confirms that SSIs are the most prevalent cause of health-care-associated infections in LMICs and that rates are more than twofold greater in developing countries than in European nations or the USA [3].

One obstacle to preventative interventions in the developing world is a lack of epidemiological data, and this problem is especially pronounced in the field of pediatric surgery, where very little is known about the incidence of SSI or about what factors may contribute to its prevalence. We have, therefore, undertaken this study to critically evaluate the incidence and putative risk factors for SSI among children at our institution in rural Kenya.

\section{The African perspective}

The incidence of pediatric SSI in LMICs ranges from 5 to $25 \%$, varying widely by region $[2,12-14]$. To our knowledge, there are only two studies that have documented rates of pediatric SSI in sub-Saharan Africa [2, 14]. In comparison to these studies, as well as to studies among adult surgical patients in sub-Saharan Africa, we have demonstrated significant improvement in SSI prevention, with an overall infection rate of $6.8 \%$ across all specialties and incision classes.

Reporting on their pediatric general surgery experience in Nigeria, Ameh and colleagues found that their patients developed SSIs in $24 \%$ of cases [2]. It should be noted, however, that the majority of patients included in their study had contaminated incisions, which presumably skewed their findings. Wound contamination is an authenticated risk for SSI, and our study simply adds to the wealth of literature which has documented wound contamination classification as an independent predisposing factor for SSI $[15,16]$.

Recently, Mwachaka and colleagues analyzed their experience with ventriculoperitoneal shunts at a large, referral hospital in Nairobi, Kenya [14]. In this study, they reported an SSI rate of $19.7 \%$ in clean cases. The factors that led to our comparatively low rate of SSI in clean cases (4.4\%) are not immediately clear. One possible explanation is our institution-wide protocol for the routine use of perioperative prophylactic antibiotics, which led to high rates of administration of antibiotics, even in clean cases.

Data regarding the implementation of peri-operative antibiotics in hospitals in LMICs are limited, and, to our knowledge, there are no trials that have focused specifically on the effect of prophylactic antibiotics in this setting. A pilot study conducted in eight hospitals participating in the World Health Organization Safe Surgery Saves Lives Challenge suggests that overall morbidity and mortality is improved with the implementation of a pre-operative checklist that includes confirmation of appropriate antibiotic choice and timing, with the rate of SSIs in participant hospitals falling from 6.2 to $3.4 \%$ [17, 18]. None of the developing world hospitals included in the study had peri- 
operative antibiotic guidelines in place, nor did they routinely use peri-operative antibiotics prior to implementation of the checklist. These data lend credence to our belief that protocol-driven use of peri-operative antibiotics mitigates the burden of SSI at our hospital and that all developing world hospitals should implement standardized prophylactic antibiotic protocols as recommended by the Center for Disease Control and the WHO [17, 19-25].

\section{The global perspective}

Although the SSI rates we have achieved compare very favorably to other African institutions, it should be remembered that great disparities exist between the lowincome and high-income nations.

In a retrospective database study of surgical patients from four American children's hospitals, an overall surgical site infection rate of $1.3 \%$ was reported with equal distribution across surgical specialties [1]. Likewise, in a retrospective, case-control study restricted to clean and clean-contaminated cases, Bucher and colleagues found that $<1 \%$ of children developed SSIs at the St. Louis Children's Hospital during a 12-year period [26]. They also identified several risk factors for surgical site infection in their patient population. Among these was the duration of operation, which was an independent risk factor for SSI in our study and has been described as such by others [2, 27, 28]. The authors did not include the duration of operation in the logistic regression analysis, but they did report that patients who required ICU admission after operation were more likely to develop SSIs. Most likely, both of these factors are actually surrogates for complexity of surgical disease and comorbidities, although SSI may be increased by intra-operative hypothermia resulting from increased duration of surgery [29].

It is also interesting to note that Bucher and colleagues discovered a racial predisposition to SSI among children [26]. In their study, children of African descent were more likely to develop SSI. The authors speculated that there may be socioeconomic factors contributing to this unexpected finding. Indeed, the obvious but ill-defined disadvantages of poverty and illiteracy are putative contributors to complications, including SSI, among pediatric patients throughout the world.

\section{Study effects and limitations}

The current study has led to several beneficial effects in our institution, in the form of measures to counteract both the resource restrictions of our hospital as well as the educational and economical disadvantages our patients face. For example, since the completion of this study, we have instituted an Infection Prevention Control (IPC) Committee for the hospital that oversees all infection prevention related measures. Among other projects, this committee has spearheaded the installation of alcohol-based, hand-rub antiseptic dispensers throughout the hospital, and they regularly educate the hospital staff on the importance of provider hand hygiene to patient care.

There has also been renewed interest in patient hygiene as a factor in SSI. Our hospital has a long-standing but inconsistently implemented protocol for bathing hospitalized children the night before and the morning of any surgical procedure, with hospitalized patients receiving freshly washed gowns and linens. Because parents typically share beds with their hospitalized children, we also require that parents use clean gowns while in the hospital. The impact of pre-operative skin disinfection by bathing has been described in the literature, but no studies of its feasibility or effectiveness have been attempted in a resource-poor setting such as ours [30]. Nonetheless, we have redoubled our efforts at improving patient hygiene prior to elective operations.

Addressing disparities such as education, nutrition, and hygiene is undoubtedly an essential part of the global SSI prevention project. Likewise, investigators should consider other regionally defined risks for SSI, such as human immune deficiency virus and the acquired immune deficiency syndrome (HIV/AIDS), which are known to predispose patients to post-operative infectious complications, including SSIs [31]. Clinical and academic resources should be directed at addressing these issues.

\section{Conclusion}

We conclude that surgical site infection remains a significant cause of morbidity among pediatric surgical patients in the developing world and that modifiable risk factors of SSI in these children remain poorly defined. While great advances have been made in the peri-operative care of children in high-income countries, major research and clinical initiatives are needed to address the basic surgical needs of children throughout low- and middle-income countries.

Acknowledgments Data were obtained as part of the clinical trial NCT00987402 for which the authors received financial support from the Velux Foundation of Switzerland through the Swiss Academy of Sciences.

\section{References}

1. Raval M, Dillon P, Bruny J et al (2011) American College of Surgeons National Surgical Quality Improvement Program Pediatric: a phase 1 report. J Am Coll Surg 212:1-11 
2. Ameh EA, Mshelbwala PM, Nasir AA et al (2009) Surgical site infection in children: prospective analysis of the burden and risk factors in a sub-Saharan African setting. Surg Infect 10:105-109

3. Allegranzi B, Nejad S, Comberscure C et al (2011) Burden of endemic health-care-associated infection in developing countries: systematic review and meta-analysis. Lancet 377:228-241

4. Nthumba P, Stepita-Poenaru E, Poenaru D et al (2010) Clusterrandomized, crossover trial of the efficacy of plain soap and water versus alcohol-based rub for surgical hand preparation in a rural hospital in Kenya. Br J Surg 97:1621-1628

5. Horan T, Gaynes R, Martone W et al (1992) CDC definitions of nosocomial surgical site infections, 1992: a modification of CDC definitions of surgical wound infections. Infect Control Hosp Epidemiol 13:606-608

6. Nichols RL (1998) Postoperative infections in the age of drugresistant Gram-positive bacteria. Am J Med 104:11S-16S

7. Haley RW, Culver DH, White JW et al (1985) The nationwide nosocomial infection rate: a new need for vital statistics. Am J Epidemiol 121:159-167

8. Hodges A, Agaba S (1997) Wound infection in a rural hospital: the benefit of a wound management protocol. Trop Doct 27:174-175

9. Wariso B, Nwachukwu C (2003) A survey of common pathogens in wound in patients at the University of Port Harcourt Teaching Hospital. West Afr J Med 22:50-54

10. Eriksen H, Chugulu S, Kondo S et al (2003) Surgical-site infections at Kilimanjaro Christian Medical Center. J Hosp Infect 55:14-20

11. Fehr J, Hatz C, Soka I et al (2006) Risk factors for surgical site infection in a Tanzanian district hospital: a challenge for the traditional National Nosocomial Infections Surveillance system index. Infect Control Hosp Epidemiol 27:1401-1404

12. Sharma LK, Sharma PK (1986) Postoperative wound infection in a pediatric surgical service. J Pediatr Surg 21:889-891

13. Duque-Estrada EO, Duarte MR et al (2003) Wound infection in pediatric surgery: a study of 575 patients in a university hospital. Pediatr Surg Int 19:436-438

14. Mwachaka P, Obonyo N, Mutiso B et al (2010) Ventriculoperitoneal shunt complications: a three-year retrospective study in a Kenyan national teaching referral hospital. Pediatr Neurosurg 46:1-5

15. Horwitz J, Chwals W, Doski J et al (1998) Pediatric wound infections: a prospective multicenter study. Ann Surg 227:553558

16. Lawal O, Adejuyigbe O, Oluwole F (1990) The predictive value of bacterial contamination at operation in post-operative wound sepsis. Afr J Med Sci 19:173-179
17. WHO (2009) Safe surgery saves lives: the second global patient safety challenge. http://www.who.int/patientsafety/safesurgery/ en/. Retrieved 18 April 2011

18. Haynes A, Weiser T, Berry W et al (2009) A surgical safety checklist to reduce morbidity and mortality in a global population. N Engl J Med 360:491-499

19. Center for Disease Control Surgical site infections (2011) Center for Disease Control and Prevention: Healthcare-associated Infections. http://www.cdc.gov/HAI/ssi/ssi.html. Retrieved 22 April 2011

20. Mangram A, Horan T, Pearson M et al (1999) Guideline for the prevention of surgical site infection, 1999. Hospital Infection Control Practices Advisory Committee. Infect Control Hosp Epidemiol 20:247-278

21. Bratzler D, Houck P (2004) Antimicrobial prophylaxis for surgery: an advisory statement for the National Surgical Infection Prevention Project. Clin Infect Dis 38:1706-1715

22. Bratzler D, Houck P, Richards C et al (2005) Use of antimicrobial prophylaxis for major surgery: baseline results from the national surgical infection prevention project. Arch Surg 140:174-182

23. Classen D, Evans R, Pestotnik SL et al (1992) The timing of prophylactic administration of antibiotics and the risk of surgicalwound infection. N Engl J Med 326:281-286

24. Dellinger E, Gross P, Barrett TL et al (1994) Quality standard for antimicrobial prophylaxis in surgical procedures. The Infectious Diseases Society of America. Infect Control Hosp Epidemiol 15:182-188

25. Dellinger E (2007) Prophylactic antibiotics: administration and timing before operation are more important than administration after operation. Clin Infect Dis 44:928-930

26. Bucher B, Guth R, Elward A et al (2011) Risk factors and outcomes of surgical site infection in children. J Am Coll Surg (in press)

27. Geubbels E, Grobbee D, Vandenbroucke-Grauls C et al (2006) Improved risk adjustment for comparison of surgical site infection rates. Infect Control Hosp Epidemiol 27:1330-1339

28. Leong G, Wilson J, Charlett A (2006) Duration of operation as a risk factor for surgical infection: comparison of English and US data. J Hosp Infect 63:255-262

29. Kurz A, Sessler DL, Lenhardt R et al (1996) Perioperative normothermia to reduce the incidence of surgical wound infection and shorten hospitalization. N Engl J Med 334:1209-1216

30. Garibaldi R, Skolnick D, Lerer T et al (1988) The impact of preoperative skin disinfection on preventing intraoperative wound contamination. Infect Control Hosp Epidemiol 9:109-113

31. Drapeau C, Pan A, Bellacosa C et al (2009) Surgical site infections in HIV-infected patients: results from an Italian prospective multicenter observational study. Infection 37:455-460 